\title{
Determinants of seasonal influenza vaccination in pregnant women in Valencia, Spain
}

\author{
R. Vila-Candel ${ }^{1,2^{*}}$ (D, P. Navarro-IIlana ${ }^{2}$, E. Navarro-Illana ${ }^{2}$, E. Castro-Sánchez ${ }^{3}$, Kiri Duke ${ }^{2}$, F. J. Soriano-Vidal ${ }^{2,4}$, \\ J. Tuells ${ }^{5}$ and J. Díez-Domingo $0^{2,6}$
}

\begin{abstract}
Background: In most countries the coverage of seasonal influenza vaccination in pregnant women is low. We investigated the acceptance, reasons for rejection and professional involvement related to vaccine information in pregnant women in Valencia, Spain.

Methods: Observational retrospective study in 200 pregnant women, 100 vaccinated and 100 unvaccinated, were interviewed during the 2014/2015 vaccination campaign. Electronic medical records, immunization registry and telephone interviews were used to determine reasons for vaccination and immunization rejection.

Results: $40.5 \%$ of pregnant women in the health department were vaccinated. The midwife was identified as source of information for $89 \%$ of women. The vaccine was rejected due to low perceptions of risk of influenza infection (23\%), lack of information (19\%), considering the vaccine as superfluous (16\%), close proximity of delivery date (13\%) and fear of side effects (12\%).

Conclusion: Pregnant women in Spain declined to be vaccinated due to under-estimation of the risk of contracting or being harmed by influenza, and lack of information. Interventions aiming to optimize vaccination coverage should include information addressing the safety and effectiveness of the current vaccine together with improved professional training and motivation.
\end{abstract}

Keywords: Influenza Vaccines, Pregnancy, Acceptance, Influenza vaccine coverage

\section{Background}

Pregnancy is an independent risk factor for developing severe seasonal influenza [1]. Many international organizations recommend the administration of influenza vaccine to pregnant women $[2,3]$ since influenza infection is associated with higher maternal morbidity and mortality, increased hospital admissions and worse perinatal outcomes $[4,5]$.

Since 2004 influenza vaccination is recommended at any stage of pregnancy due to the well documented safety profile of the vaccine [6]. Despite its benefits, vaccination coverage among pregnant women remains low

\footnotetext{
* Correspondence: rvila@hospital-ribera-com

'Midwifery at La Ribera Hospital Health Department, Carretera Corbera, km 1, 46600 Alzira, Valencia, Spain

2Faculty of Nursing, Universidad Católica de Valencia" San Vicente Mártir", c/ Espartero, 7. 46007 Valencia, Spain

Full list of author information is available at the end of the article
}

[7], according to international studies. In Spain there are no published data on influenza vaccination coverage in pregnant women, despite the clear benefits derived from close epidemiological surveillance of such coverage [8].

Different authors $[1,9]$ have highlighted that vaccination recommendation by health professionals is the main reason why women choose to be vaccinated against influenza. However, lack of information by health professionals also remains a frequently cited determining factor for rejecting vaccination [10]. Other studies have identified additional influences such as emotional or psychological factors (subjective emotional experiences, e.g. fear of side effects, doubts about the effectiveness of the vaccine, fear of needles/pain etc.) or under-estimation of personal risk (beliefs about the limited severity of the illness, influenza vaccination being unimportant, or non- 
association with important sequelae) [11-13] contributing to low vaccination rates.

Since a variety of studies have explored the scope of professional advice and its impact on vaccination acceptance, such evidence could be used to inform optimal strategies to improve vaccination coverage in this population at-risk [3, 14-16]. In Spain, pregnant women are entitled to receive free influenza vaccination.

The aim of the study was to investigate the acceptance of influenza vaccination amongst pregnant women in Valencia (Spain) and the reasons expressed for vaccination rejection. We also analyzed the knowledge that pregnant women had about the vaccine and described which healthcare professional was more frequently involved in the vaccination decision-making process during pregnancy.

\section{Methods}

We conducted an observational, descriptive, retrospective study in women seen at La Ribera healthcare department in Valencia (Spain) for pregnancy follow-up and delivery between October 1, 2014 and January 31, 2015. This healthcare department has a La Ribera University Hospital (HULR), which is a tertiary healthcare center with 300 beds. The hospital and the healthcare department provide health services to 250,000 people approximately.

In the community (or primary care) we have family doctors, community nurses, community midwives and gynecologists as baseline care providers. One provider complements the other provider's services and pregnant patients are followed up by the family doctor, the community midwife and the gynecologist.

During the study period there were 644 deliveries, of those 100 were not resident of the catchment area and 124 there was no information about the vaccination status in the vaccine registry Sistema de Información Vacunal (SIV) [17].

Of the remaining 420 women, 170 (40.5\%) had received influenza vaccination. After reviewing the electronic charts we excluded women under 18 years, allergic to any of the vaccine components or women with communication barriers.

Of the remaining, we selected 100 women to be interviewed for each group (vaccinated and unvaccinated) through simple randomization sampling. Women were contacted by telephone in Feb-March 2015, and were interviewed after accepting a verbal informed consent.

We conducted a three-question telephone survey and asked vaccinated women about the source of the information of influenza vaccine, the health provider involved in recommending the vaccination, and whether they would opt to be vaccinated again in their next pregnancy (Table 1).
Table 1 Telephone questionnaire for vaccinated and unvaccinated women

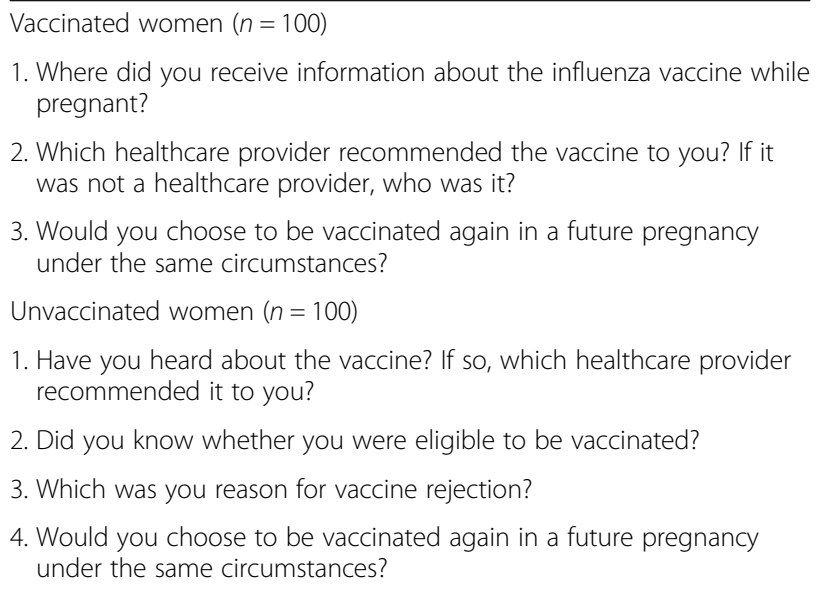

We also conducted a four-question telephone survey on unvaccinated women, where we asked whether they had heard about the vaccine and, if so, which healthcare provider recommended it, the reasons for vaccine rejection, their knowledge about the vaccine and whether they would have the vaccine given in their next pregnancy (Table 1).

Other variables collected from the interview and the review of the electronic clinical notes included age, country of origin, gestational age at delivery, parity, and chronic underlying disease (asthma, diabetes, heart disease and immunosuppression).

The sample size was calculated to assess differences in the percentage of pregnant women that received advice from the health provider for influenza vaccination. We estimated that $50 \%$ of vaccinated women received advice and $40 \%$ of the unvaccinated. With an error of $5 \%$ and a power of $85 \%$, the sample size needed was 194 women in total, 97 per group.

Bivariate analysis was performed using Chi-square; for risk factors for vaccination, an odds ratio (OR) with a 95\% CI, was calculated. The significance level was set at $p<0.05$.

\section{Ethics}

The study was conducted according to the Declaration of Helsinki and current legislation, and received approval by the HULR Research Ethics Committee and the Spanish Medicines and Medical Devices Agency.

\section{Results}

We contacted 141 vaccinated pregnant women, of those 41 (29\%) did not participate: 35 (85.3\%) did not answer the telephone, 4 (9.8\%) declined to participate in the study and $2(4.9 \%)$ were excluded due to communication difficulties. All of the vaccinated pregnant women 
confirmed their vaccination status, in agreement with the information on SIV.

On the other hand, 161 unvaccinated women were also contacted and 61 (37.9\%) did not participate: 56 (91.8\%) did not answer the telephone, 4 (6.6\%) did not accepted participation and 1 (1.6\%) was excluded due to communication barriers. All of the women with no information about the vaccination status in SIV confirmed having not received the vaccine.

The mean age was $31.5 \pm 5.2$ years (median 32.50, range 18-42), mean gestational age at delivery was $39.14 \pm 1.5$ weeks (range 34-42), and $47.2 \%(94 / 200)$ women were primiparous. The mean gestational age of vaccinated women was $32.28 \pm 3.7$ weeks (range 25-39).

The characteristics of both groups, vaccinated and unvaccinated are shown in Table 2, showing no statistical differences between them. Only $23 \%(3 / 13)$ of women with comorbidities (5.5\% asthma [11/200]; 0.5\% diabetes [1/200]; $0.5 \%$ cardiac pathology [1/200]) were vaccinated, and $36 \%(36 / 100)$ women with at-risk pregnancy received vaccination. We observed that $40 \%(6 / 15)$ of the group who received the vaccine during the previous season (2013-14) rejected the vaccination during the pregnancy.

Table 2 Characteristics of pregnant women in study $(N=200)$

\begin{tabular}{|c|c|c|c|c|c|c|}
\hline & & Vacci & & Unva & & \\
\hline & & $\overline{(n=1}$ & & $\overline{(n=}$ & & \\
\hline & & $\bar{n}$ & $\%$ & $\bar{n}$ & $\%$ & $P$-value \\
\hline Country of Origin & Foreign & 20 & 20.0 & 21 & 21.0 & \\
\hline & Spanish & 80 & 80.0 & 79 & 79.0 & 0.831 \\
\hline Age (years) & $<25$ & 18 & 18.0 & 9 & 9.0 & \\
\hline & $25-35$ & 63 & 63.0 & 66 & 66.0 & \\
\hline & $>35$ & 19 & 19.0 & 25 & 25.0 & 0.143 \\
\hline Parity & 1 & 51 & 51.0 & 43 & 43.0 & \\
\hline & 2-mar & 38 & 38.0 & 50 & 50.0 & \\
\hline & $>3$ & 11 & 11.0 & 7 & 7.0 & 0.201 \\
\hline Gestational & $<37$ & 9 & 9.0 & 10 & 10.0 & \\
\hline Age (weeks) & $37-42$ & 88 & 88.0 & 88 & 88.0 & \\
\hline & $>42$ & 3 & 3.0 & 2 & 2.0 & 0.881 \\
\hline Asthma $^{\mathrm{b}}$ & No & 97 & 97.0 & 92 & 92.0 & \\
\hline & Yes & 34.0 & 3.0 & 8 & 8.0 & 0.121 \\
\hline Diabetes Mellitus ${ }^{b}$ & No & 100 & 100.0 & 99 & 99.0 & \\
\hline & Yes & 0 & 0.0 & 1 & 1.0 & 0.316 \\
\hline Cardiac Pathology ${ }^{b}$ & No & 100 & 100.0 & 99 & 99.0 & \\
\hline & Yes & 0 & 0.0 & 1 & 1.0 & 0.316 \\
\hline Immunosuppression $^{\text {b }}$ & No & 100 & 100.0 & 100 & 100.0 & \\
\hline & Yes & 0 & 0.0 & 0 & 0.0 & N/A \\
\hline Low Risk Pregnancy ${ }^{c}$ & No & 34 & 34.0 & 40 & 40.0 & \\
\hline & Yes & 66 & 66.0 & 60 & 60.0 & 0.380 \\
\hline Pregnancy-induced Hypertension ${ }^{c}$ & No & 96 & 96.0 & 97 & 97.0 & \\
\hline & Yes & 4 & 4.0 & 3 & 3.0 & 0.700 \\
\hline Thyroid Disease $^{c}$ & No & 92 & 92.0 & 89 & 89.0 & \\
\hline & Yes & 8 & 8.0 & 11 & 11.0 & 0.469 \\
\hline Smoker status ${ }^{c}$ & No & 84 & 84.0 & 79 & 79.0 & \\
\hline & Yes & 16 & 16.0 & 21 & 21.0 & 0.363 \\
\hline Vaccinated previous season (2013-14) & No & 91 & 91.0 & 94 & 94.0 & \\
\hline & Yes & 9 & 9.0 & 6 & 6.0 & 0.421 \\
\hline
\end{tabular}

${ }^{a} P$-value: based on chi-square test; N/A: insufficient cell number to perform chi-square analysis

b Pre-gestational disease

c Gestational disease or status during pregnancy 


\section{Influenza vaccine acceptance}

When vaccinated women were asked about the source of information regarding the vaccine, 98\% (98/100) recalled being informed in their primary health care center. $1 \%$ knew about it in advance as they were health professionals and another $1 \%$ received information via mass media such as radio, television or public health communication campaigns.

The information and recommendation of vaccination came mainly from their midwives (89\%), in 9\% (9/100) from the family doctor and $2 \%$ of women did not provide any information.

Of the vaccinated, $99 \%$ would have the influenza vaccine again in a future pregnancy should they find themselves in comparable health conditions.

\section{Influenza vaccine rejection}

$14 \%$ of women had not heard of influenza vaccination during pregnancy. Those who did have information had mainly received it from their midwife $(n=40)$ or other sources such as their family doctor $(n=6)$ or gynecologist $(n=1)$. Other information channels included public health advertising campaigns $(n=12)$, relatives or friends $(n=14)$ or at work $(n=2)$. Of the total sample, $6 \%(12 / 200)$ were health care professionals and knew about the vaccine, however, $91.6 \%(11 / 12)$ of these women declined to be vaccinated $[\mathrm{OR}=13.1,95 \% \mathrm{CI}$ : $1.6-102.6 ; p=0.02]$.
When asked if they were eligible to be vaccinated, $59 \%$ (59/100) responded positively, $4 \%$ (4/100) recognized they could not receive it at the time and 12\% (12/100) did not know.

The reasons provided by women to decline the influenza vaccine during pregnancy are presented in Fig. 1. Women also stated that the main reason for rejecting vaccination was under-estimation of the risk, reflected in perceptions of no risk of contracting the illness whilst being pregnant (23\%), followed by a lack of information (19\%), considering the vaccine as non-essential (16\%) and avoiding it due to the close delivery date (13).

When women were asked if they would opt to be vaccinated in a future pregnancy should they remain in similar health condition, 18\% (18/100) accepted, 65\% $(65 / 100)$ would again reject it and $17 \%(17 / 100)$ did not know what to answer.

\section{Discussion}

This study suggests that pregnant women do not vaccinate against influenza due to the low perception of risk and a lack of evidenced-based information provided by health professionals.

No significant differences were identified in the socio-demographic and obstetric characteristics between vaccinated and unvaccinated pregnant women. Seasonal influenza remains a global public health problem [18] and demands specific strategies and a

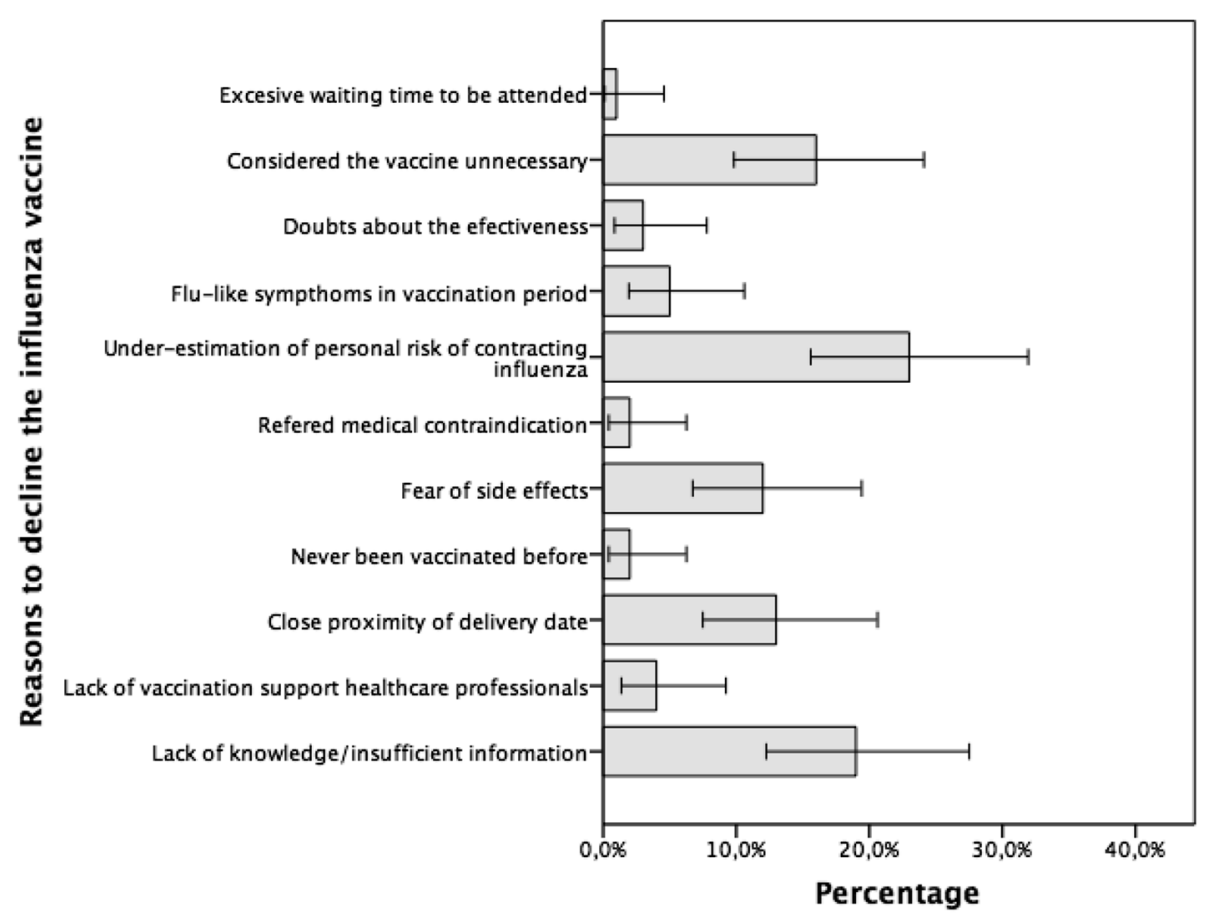

Error bars: $95 \%$ confidence interval

Fig. 1 Reasons for influenza vaccine rejection during pregnancy 
particular focus on pregnant women as a high-risk group for seasonal flu $[19,20]$.

\section{Influenza vaccine acceptance}

Regarding vaccination status before pregnancy, we observed that having previously received the flu vaccination was positively associated to acceptance during pregnancy [21]. However, a medical history involving a risk prior to pregnancy, such as having asthma, diabetes or a heart disease during pregnancy, was not associated with a higher influenza vaccination rate.

In relation to the knowledge of women who reported having received information about the vaccine, both vaccinated and unvaccinated women agreed that the midwife had been the main source of information, in agreement with a similar study conducted in the UK [22]. Although in other countries the family doctor has been reported as the main information agent [2, 16, 23], possibly owing to different healthcare models.

Concerning the intention to being re-vaccinated in a future pregnancy were the women to be in the same conditions, the surveyed women presented mixed responses. Virtually all vaccinated women agreed they would be vaccinated as well, while, in contrast, those unvaccinated would decline, coinciding with certain studies $[24,25]$. As for unvaccinated pregnant women, we observed that those who were healthcare professionals were paradoxically much more likely to decline to be vaccinated, as seen in other studies $[2,26]$.

In all, reasons for vaccination rejection can be classified according to four themes:

1) Lack of knowledge and information The lack of knowledge due to insufficient information (19\%), together with a lack of vaccination support from healthcare professionals (4\%), accounted for $23 \%$ of vaccination refusal reasons, corroborating previous results [2, 10, 27, 28]. These findings highlight the need to improve influenza vaccination promotion activities conducted by healthcare professionals for pregnant patients [3]. We believe that information should be timely provided, within a meaningful discussion and a shared decision-making perspective $[9,11]$ that favors vaccine acceptance or rejection within an optimum time frame [25]. Ideally, this discussion should reflect the information on the causes, symptoms, side effects and the importance of the disease to support their decisions.

However, the provision of accurate and quality information would be insufficient to ensure adequate health outcomes if the influence of health literacy of patients is not accounted for. Health literacy links knowledge and user skills in appropriate decision making related to health and social care [29]. A low health literacy level has been linked to poor health outcomes, including low vaccination rates [30]. In our study, unvaccinated women had accessed a greater number of information channels, compared with vaccinated women. This difference possibly reflects a need to confirm personal perceptions about the vaccine that would not fit or be accepted by the mainstream vaccination narrative [31], or that are not in agreement with the information received from each channel [25].

Therefore, in order to increase the number of vaccinated pregnant women, public health strategies could take advantage of intervention synergies, combining efficient communication materials with information regarding immunization and vaccine safety, along with continuous education strategies for healthcare professionals. Since midwives already achieve the highest rate of influenza vaccination recommendation, and as main source of information according to women $[9,24]$, their leadership on the design, implementation and evaluation of these multiple interventions would seem logical [29].

2) Pregnant women feel the vaccine is unnecessary, ineffective and that they have a low perception of risk of influenza infection

$16 \%$ of women surveyed considered the vaccine unnecessary. Such idea may be supported by considering that the risk of contracting the disease whilst pregnant is non existent (23\%). Pregnant women could also assume that an absence of influenza infection in the pre-pregnancy period could be related to their lifestyle, and since they had not changed it, the infection would also not occur during pregnancy either. Additionally, 3\% of the women considered the vaccine to be ineffective, failing to realize the wider effect that an insufficient number of vaccinated women would have on the effectiveness of the vaccine at population level [32]. Furthermore, $13 \%$ of surveyed women believed that vaccination was unnecessary due to the imminent childbirth date. Such position obviously ignores the benefits that acquired immunity would provide for the newborn after delivery [27], and that may result in a significant reduction in perinatal infections [6].

3) Health risk perceptions

The side effects of the vaccine were a concern for $12 \%$ of women. This apprehension referred not only to their own health but also the babies', and would explain the lack of vaccine acceptance during pregnancy. Such negative perceptions, however, do not seem to be endorsed by the existing on the link between vaccination and adverse perinatal and maternal outcomes [8, 32-34]. 
In short, our results consistently confirm those obtained by other groups $[1,27,35]$, where more than half of vaccination rejections stemmed from a perceived lack of data on the efficacy and safety of vaccine. Due to the determinant influence of these perceptions and beliefs, it is essential to carry out qualitative studies that would inform and facilitate improvement interventions.

4) Medical contraindications

In $2 \%(2 / 100)$ of the cases the vaccine could not be administered due to transient and self-limited infectious processes, simply requiring a postponement of the inoculation [31, 32].

\section{Conclusions}

Pregnant women in Spain declined to be vaccinated due to an under-estimation of the risk of contracting or being harmed by influenza and lack of information. In order to increase the acceptance of influenza vaccine it would be necessary to improve the information offered to women by all members of the multidisciplinary team and to integrate the therapeutic advice onto the care path for pregnant women.

\section{Abbreviations}

HULR: Hospital Universitario de la Ribera; SIV: Sistema de Información vacunal (vaccination registry)

\section{Acknowledgements}

We are grateful to all the staff at the hospital and health facilities of La Ribera University Hospital, and their support with this research.

\section{Funding}

This project has been funded by a grant awarded by Hospital Universitario de la Ribera PBR24/15. Enrique Castro-Sánchez is affiliated to the National Institute for Health Research Health Protection Research Unit (NIHR HPRU) in Healthcare Associated Infection and Antimicrobial Resistance at Imperial College London, in partnership with Public Health England. The views expressed are those of the authors and not necessarily those of the NHS, the $\mathrm{NIHR}$, the Department of Health, or Public Health England. ECS has received an Early Career Research Fellowship from the Antimicrobial Research Collaborative at Imperial College London, and acknowledges the support of the Florence Nightingale Foundation.

\section{Availability of data and material}

The original dataset is available from the corresponding author on reasonable request.

\section{Authors' contributions}

RVC drafted the manuscript. JDD, PNI, FJSV and JT were involved in conceptualizing the study. RVC, PNI and ECS conducted the data analyses. All authors were involved in writing the manuscript and approve of its final version.

\section{Competing of interests}

The authors declare that they have no competing of interests.

\section{Consent for publication}

Not applicable.

\section{Ethics approval and consent to participate}

Participants provided informed consent to participate in the study, and the Human Ethics Committee at Hospital Universitario de La Ribera Research
Ethics Committee and Research Commission and the Spanish Medicines and Medical Devices Agency approved the study (HULR15/12-01).

\section{Author details}

${ }^{1}$ Midwifery at La Ribera Hospital Health Department, Carretera Corbera, km 1, 46600 Alzira, Valencia, Spain. ${ }^{2}$ Faculty of Nursing, Universidad Católica de Valencia" San Vicente Mártir", c/ Espartero, 7. 46007 Valencia, Spain. ${ }^{3}$ National Institute for Health Research Health Protection Research Unit (NIHR HPRU) In Healthcare Associated Infection and Antimicrobial Resistance at Imperial College London, Du Cane Road, London W12 ONN, UK. ${ }^{4}$ Xàtiva-Ontinyent Health Department, Ausias March, 46.800 Xativa, Spain. ${ }^{5}$ Cátedra de Vacunología Balmis, University of Alicante, Carretera de San Vicente del Raspeig, s/n. 03690, San Vicente del Raspeig, Alicante, Spain. 'Vaccine Research, Fundación para el Fomento de la Investigación Sanitaria y Biomédica de la Comunitat Valenciana, FISABIO-Public Health, Avda. Catalunya, 21. 46020 Valencia, Spain.

Received: 18 August 2016 Accepted: 4 November 2016

Published online: 21 November 2016

\section{References}

1. Yuen CYS, Tarrant M. A Comprehensive Review of Influenza and Influenza Vaccination During Pregnancy. J Perinat Neonatal Nurs. 2014;28:261-70.

2. Panda B, Stiller R, Panda A. Influenza vaccination during pregnancy and factors for lacking compliance with current CDC guidelines. J Matern Fetal Neonatal Med. 2011;24:402-6. doi:10.3109/14767058.2010.497882.

3. Yuen CYS, Tarrant M. Determinants of uptake of influenza vaccination among pregnant women - A systematic review. Vaccine. 2014;32:4602-13. doi:10.1016/j.vaccine.2014.06.067.

4. McNeil SA, Dodds LA, Fell DB, Allen VM, Halperin BA, Steinhoff MC, et al. Effect of respiratory hospitalization during pregnancy on infant outcomes. Am J Obstet Gynecol 2011;204. doi:10.1016/j.ajog.2011.04.031.

5. Hartert TV, Neuzil KM, Shintani AK, Mitchel EF, Snowden MS, Wood LB, et al. Maternal morbidity and perinatal outcomes among pregnant women with respiratory hospitalizations during influenza season. Am J Obstet Gynecol. 2003;189:1705-12. doi:10.1016/50002-9378(03)00857-3.

6. Munoz FM. Safety of influenza vaccines in pregnant women. Am J Obstet Gynecol 2012;207. doi:10.1016/j.ajog.2012.06.072.

7. Lu P, Bridges $C B$, Euler $G L$, Singleton JA. Influenza vaccination of recommended adult populations, U.S., 1989-2005. Vaccine. 2008;26:1786-93. doi:10.1016/j.vaccine.2008.01.040.

8. Vilca Yengle LM, Campins Martí M, Cabero Roura L, Rodrigo Pendás JÁ, Martínez Gómez X, Hermosilla Pérez E, et al. Influenza vaccination in pregnant women. Coverage, practices and knowledge among obstetricians. Med Clin (Barc). 2010;134:146-51.

9. Regan AK, Mak DB, Hauck YL, Gibbs R, Tracey L, Effler P V. Trends in seasonal influenza vaccine uptake during pregnancy in Western Australia: Implications for midwives. Women Birth 2016. doi:10.1016/j. wombi.2016.01.009

10. Dubé E, Laberge C, Guay M, Bramadat P, Roy R, Bettinger JA. Vaccine hesitancy: An overview. Hum Vaccin Immunother. 2013;9(8):1763-73. doi:10.4161/hv.24657.

11. Lester RT, McGeer A, Tomlinson G, Detsky AS. Use of, effectiveness of, and attitudes regarding influenza vaccine among house staff. Infect Control Hosp Epidemiol. 2003;24:839-44. doi:10.1086/502146.

12. Ofstead CL, Tucker SJ, Beebe TJ, Poland GA. Influenza vaccination among registered nurses: information receipt, knowledge, and decision-making at an institution with a multifaceted educational program. Infect Control Hosp Epidemiol. 2008;29:99-106. doi:10.1086/526431.

13. Yaqub O, Castle-Clarke S, Sevdalis N, Chataway J. Attitudes to vaccination: A critical review. Soc Sci Med. 2014;112:1-11. doi:10.1016/j. socscimed.2014.04.018.

14. Perlin JB, Septimus EJ, Cormier SB, Moody JA, Hickok JD, Bracken RM. Developing a program to increase seasonal influenza vaccination of healthcare workers: lessons from a system of community hospitals. J Healthc Qual. 2013;35:5-15. doi:10.1111/jhq.12005.

15. Henninger M, Naleway A, Crane B, Donahue J, Irving S. Predictors of seasonal influenza vaccination during pregnancy. Obstet Gynecol. 2013;121:741-9. doi:10.1097/AOG.0b013e3182878a5a.

16. Privileggio L, Falchi A, Grisoni M-L, Souty C, Turbelin C, Fonteneau L, et al. Rates of immunization against pandemic and seasonal influenza in persons 
at high risk of severe influenza illness: a cross-sectional study among patients of the French Sentinelles general practitioners. BMC Public Health. 2013;13:246. doi:10.1186/1471-2458-13-246.

17. Pérez-Vilar S, Díez-Domingo J, López-Lacort M, Martínez-Úbeda S, MartinezBeneito MA. Effectiveness of rotavirus vaccines, licensed but not funded, against rotavirus hospitalizations in the Valencia Region, Spain. BMC Infect Dis. 2015;15:92. doi:10.1186/s12879-015-0811-5.

18. Puig-Barberá J, Tormos A, Trushakova S, Sominina A, Pisareva M, Ciblak MA, et al. The Global Influenza Hospital Surveillance Network (GIHSN): A new platform to describe the epidemiology of severe influenza. Influenza Other Respi Viruses. 2015;9:277-86. doi:10.1111/irv.12335.

19. Stockwell MS, Westhoff C, Kharbanda EO, Vargas CY, Camargo S, Vawdrey DK, et al. Influenza vaccine text message reminders for urban, low-income pregnant women: A randomized controlled trial. Am J Public Health 2014;104. doi:10.2105/AJPH.2013.301620.

20. France EK, Smith-Ray R, McClure D, Hambidge S, Xu S, Yamasaki K, et al. Impact of maternal influenza vaccination during pregnancy on the incidence of acute respiratory illness visits among infants. Arch Pediatr Adolesc Med. 2006;160:1277-83.

21. Gorman JR, Brewer NT, Wang JB, Chambers CD. Theory-based predictors of influenza vaccination among pregnant women. Vaccine. 2012;31:213-8. doi:10.1016/j.vaccine.2012.10.064.

22. Ishola DA, Permalloo N, Cordery RJ, Anderson SR. Midwives' influenza vaccine uptake and their views on vaccination of pregnant women. J Public Health (United Kingdom). 2013;35:570-7. doi:10.1093/pubmed/fds109.

23. Laenen J, Roelants M, Devlieger R, Vandermeulen C. Influenza and pertussis vaccination coverage in pregnant women. Vaccine. 2015;33:2125-31. doi:10. 1016/j.vaccine.2015.03.020

24. Meharry PM, Colson ER, Grizas AP, Stiller R, Vázquez M. Reasons why women accept or reject the trivalent inactivated influenza vaccine (TIV) during pregnancy. Matern Child Health J. 2013;17:156-64. doi:10.1007/ s10995-012-0957-3.

25. Maher L, Dawson A, Wiley K, Hope K, Torvaldsen S, Lawrence G, et al. Influenza vaccination during pregnancy: a qualitative study of the knowledge, attitudes, beliefs, and practices of general practitioners in Central and South-Western Sydney. BMC Fam Pract. 2014;15:102. doi:10.1186/1471-2296-15-102.

26. Torner N, Godoy P, Soldevila N, Toledo D, Rius C, Domínguez A. Primary health care professionals attitudes towards influenza immunzation in Catalonia. Aten Primaria. 2015;48:192-9. doi:10.1016/j.aprim.2014.09.014.

27. Mak TK, Mangtani P, Leese J, Watson JM, Pfeifer D. Influenza vaccination in pregnancy: current evidence and selected national policies. Lancet Infect Dis. 2008;8:44-52.

28. Falomir-Pichastor JM, Toscani L, Despointes SH. Determinants of flu vaccination among nurses: The effects of group identification and professional responsibility. Appl Psychol. 2009;58:42-58. doi:10.1111/j.14640597.2008.00381.X.

29. Castro-Sánchez E, Chang PWS, Vila-Candel R, Escobedo AA, Holmes AH. Health literacy and infectious diseases: why does it matter? Int J Infect Dis. 2016:43:103-10. doi:10.1016/j.jij. 2015.12.019.

30. Berkman ND, Sheridan SL, Donahue KE, Halpern DJ, Crotty K. Low health literacy and health outcomes: An updated systematic review. Ann Intern Med. 2011;155:97-107. doi:10.7326/0003-4819-155-2-201107190-00005.

31. Yudin MH, Salaripour M, Sgro MD. Pregnant women's knowledge of influenza and the use and safety of the influenza vaccine during pregnancy. J Obstet Gynaecol Can. 2009;31:120-5. doi:10.1016/S1701-2163(16)34095-6.

32. Tamma PD, Ault KA, Del Rio C, Steinhoff MC, Halsey NA, Omer SB. Safety of influenza vaccination during pregnancy. Am J Obstet Gynecol. 2009;201:547-52.

33. Kharbanda EO, Vazquez-Benitez G, Lipkind H, Naleway A, Lee G, Nordin JD. Inactivated influenza vaccine during pregnancy and risks for adverse obstetric events. Obstet Gynecol. 2013;122:659-67. doi:10.1097/AOG. 0b013e3182a1118a.

34. Nordin JD, Kharbanda EO, Vazquez Benitez G, Lipkind H, Vellozzi C, DeStefano F. Maternal Influenza Vaccine and Risks for Preterm or Small for Gestational Age Birth. J Pediatr. 2016;164:1051-7.e2. doi:10.1016/j.jpeds.2014.01.037.

35. Christian LM. Optimizing benefits of influenza virus vaccination during pregnancy: Potential behavioral risk factors and interventions. Vaccine. 2014;32:2958-64.

\section{Submit your next manuscript to BioMed Central and we will help you at every step:}

- We accept pre-submission inquiries

- Our selector tool helps you to find the most relevant journal

- We provide round the clock customer support

- Convenient online submission

- Thorough peer review

- Inclusion in PubMed and all major indexing services

- Maximum visibility for your research

Submit your manuscript at www.biomedcentral.com/submit 